\title{
Minijobs im Bäckerhandwerk und Gastgewerbe
}

\author{
KARSTEN ROTHE, PETER STÖRLING, GUIDO ZEITLER
}

Durch die starke Zunahme von Minijobs haben sich die Beschäftigungsstrukturen im Organisationsbereich der Gewerkschaft Nahrung-Genuss-Gaststätten (NGG) nachhaltig verändert. Dies gilt besonders für das Backgewerbe und das Gastgewerbe, wo die Anteile der geringfügigen Beschäftigung besonders hoch sind: Im Backgewerbe ist knapp jeder dritte Arbeitsplatz ein Minijob, im Gastgewerbe ist es mittlerweile jeder zweite. Die betriebliche Praxis zeigt, dass sich die Beschäftigungsbedingungen mit wachsenden Anteilen von Minijobs in den beiden Branchen verschlechtert haben: Die Arbeitszeiten werden insbesondere bei den Minijobs dem Arbeitsanfall kurzfristig angepasst, unbezahlte Mehrarbeit ist im Minijob keine Ausnahme und tarifliche und arbeitsrechtliche Standards werden auch nicht eingehalten.

\section{Minijobs im Backgewerbe}

Das Backgewerbe ist in der Ernährungsindustrie die Branche mit der höchsten Beschäftigtenanzahl und den meisten Betrieben. Backwaren werden nach wie vor in einer großen Zahl von Klein- und Mittelbetrieben hergestellt, deren Ursprung im Bäckerhandwerk liegt. Jedoch macht der Strukturwandel auch vor dem Backgewerbe nicht halt. Die Konkurrenz mit dem Lebensmitteleinzelhandel und sich wandelnde Verbrauchergewohnheiten haben einen Verdrängungswettbewerb initiiert, in dessen Folge die Zahl der Betriebe abnimmt und die Betriebe tendenziell größer werden. Damit ändern sich Beschäftigungsumfang und Beschäftigungsbedingungen. Bei stark rationalisierten Produktionsstrukturen arbeitet in der Herstellung von Backwaren nur noch ein kleiner Teil der Beschäftigten, der überwiegende Teil - in der Mehrheit Frauen - arbeitet im Verkauf, vor allem in Filialbetrieben.

Von den rund 310.000 Beschäftigten im Backgewerbe sind 220.000 Personen sozialversicherungspflichtig beschäftigt. Sozialversicherungspflichtige Beschäftigung im Backgewerbe war in den vergangenen Jahren bei Frauen und Männern gleichermaßen rückläufig. Der Anteil der Teilzeitbeschäftigten ist von 2000 bis 2007 um knapp 3,8 Prozentpunkte (ca. 1.170 Personen) gestiegen. Im gleichen Zeitraum ist der Anteil der sozialversicherungspflichtigen Vollzeitbeschäftigten um mehr als 10 Prozentpunkte gesunken (ca. 24.500 Personen). Parallel zur ansteigenden Teilzeitbeschäftigung stieg auch die Zahl der Minijobs. 30\% bzw. 90.000 aller Beschäftigten sind Minijobber. Von den sozialversicherungspflichtig Beschäftigten sind fast zwei Drittel Frauen, im Bereich der geringfügigen Beschäftigung liegt der Frauenanteil bei über $80 \%$.

Teilzeitarbeit ist insbesondere im Verkauf des Bäckerhandwerks verbreitet. Dieses geschieht meist nicht freiwillig! In der betrieblichen Praxis wird Vollzeitbeschäftigung von Frauen im Verkauf in „lange“ Teilzeitbeschäftigung, (z. B. 120 Stunden im Monat) umgewandelt. Die tatsächliche Arbeitszeit ist aber häufig höher als die vereinbarte, wobei Mehrarbeitszuschläge in der Regel nicht gezahlt werden. Vorbereitende Arbeiten vor Ladenöffnung werden erst gar nicht zur Arbeitszeit gerechnet, dies wird als Selbstverständlichkeit vorausgesetzt. Die Betriebe erwarten von den Verkäuferinnen in Teilzeit eine höhere Einsatzflexibilität. Sie dient den Unternehmen dazu, den erweiterten Öffnungszeiten gerecht zu werden und richtet sich äußerst selten nach den Bedürfnissen der Beschäftigten.

Bäckereien in sogenannten „Vorkassenzonen“ von Einzelhandelsbetrieben richten die Verkaufszeit in der Regel nach der Ladenöffnungszeit des Einzelhandels aus. Der Verkauf von Backwaren nach 18.00 Uhr ist dabei nicht immer besonders gewinnbringend. In den Abendstunden wie auch zu Stoßzeiten werden vielfach geringfügig Beschäftigte eingesetzt. Es gibt keine genaue Festlegung der Stundenzahl in den Arbeitsverträgen der Minijobber. Dies führt in der Praxis dazu, dass von ihnen verlangt wird, solange zu bedienen bis der Tresen leer ist bzw. bis der große Kundenansturm vorbei ist. Die Stoßzeiten sind je nach Standort der Bäckereien unterschiedlich. In der Nähe von Schulen oder in der Nähe von Firmen oder Büros ist der Arbeitsanfall in der Mittagszeit besonders hoch, während er sich an anderen Standorten anders verteilen kann. Auf Nachfragen unserer Kolleginnen, wie es mit Sonderzahlungen aufgrund von längeren Arbeitszeiten sei, verweisen die Betriebe auf die Verdiensthöchstgrenze von maximal $400 €$ in geringfügigen Beschäftigungsverhältnissen und verweigern die Zahlung der tatsächlich geleisteten Stunden. Durch die effektive Stundenzahl ergibt sich dann eine Bezahlung deutlich unterhalb des tariflichen Entgeltniveaus.

Wir würden es noch nicht als Trend bezeichnen, aber zu beobachten ist auch, dass Vollzeit-Verkäuferinnen mit einer Arbeitszeit von Montag bis Samstag mit einem zusätzlichen Minijob-Vertrag am Sonntag arbeiten. Aus rechtlichen Gründen meist nicht beim selben Arbeitgeber, sondern der Arbeitsvertrag wird mit eigens hierfür gegründeten 
Tochterunternehmen abgeschlossen. Dies wird den Beschäftigten durch die Befreiung von Sozialversicherungsbeiträgen im Minijob besonders attraktiv gemacht und auch so von den Arbeitgebern angeboten: Die Überstunden am Sonntag werden im Minijob brutto gleich netto ausgezahlt.

Ebenso ist zu beobachten, dass die Tätigkeit der Belieferung von Bäckereifilialen zunehmend im Minijob ausgeübt wird, obwohl arbeitsorganisatorisch auch ein sozialversicherungspflichtiges Beschäftigungsverhältnis mit einer höheren Wochenstundenzahl möglich wäre. Diese Tätigkeit als sogenannte „Frischdienstfahrer“ scheint im Minijob gerade für männliche Rentner interessant zu sein, die dadurch ihre Rente aufbessern. Insgesamt liegt der Anteil von über 50-jährigen geringfügig Beschäftigten im Backgewerbe bei einem Drittel. Das ist möglicherweise ein Hinweis auf niedrige Renten als Folge von niedrigen Löhnen in der Erwerbsphase, die einen Hinzuverdienst erfordern. Andere Tourenfahrer arbeiten im Minijob als Nebentätigkeit, was auf ein niedriges Entgelt im Hauptjob, also dem sozialversicherungspflichtigen Beschäftigungsverhältnis, hindeuten kann.

\section{Minijobs im Gastgewerbe}

Im Gastgewerbe ist fast jedes zweite Beschäftigungsverhältnis ein Minijob. Die geringfügige Beschäftigung ist in dieser Branche mit insgesamt rund 1,6 Mio. Beschäftigten daher fast zu einer Art „Normalarbeitsverhältnis“ geworden und hat insbesondere seit der gesetzlichen Neuregelung im Jahr 2003 deutlich zugenommen: Laut Beschäftigungsstatistik der Bundesagentur für Arbeit (BA) gab es im Gastgewerbe im Dezember 2010 rund 792.000 geringfügige Beschäftigungsverhältnisse gegenüber 822.209 sozialversicherungspflichtigen Beschäftigungsverhältnissen. Im Vergleich zu Juni 2004, also gut einem Jahr nach der Reform der geringfügigen Beschäftigung, stieg der Minijobanteil um 24\%. Besonders stark war das Wachstum der im Nebenjob geringfügig entlohnten Beschäftigten: Ihr Anteil stieg von 183.000 auf 268.000 Personen. In der betrieblichen Praxis ist zu beobachten, dass vor allem sozialversicherungspflichtige Vollzeitstellen durch Minijobs und andere Teilzeitarbeitsverhältnisse verdrängt werden. Als Ursachen für diese Entwicklung sind mehrere Aspekte auszumachen, die nun näher beleuchtet werden.

Im Gastgewerbe und insbesondere im Gaststättenbereich sind der überwiegende Teil der knapp 176.000 Betriebe Klein- und Kleinstbetriebe. Diese Situation spiegelt sich auch in den Beschäftigtenzahlen wider. In Großunternehmen mit mehr als 500 Beschäftigten arbeiteten nur etwas mehr als $7 \%$ der Beschäftigten. Neun von zehn Gastgewerbeunternehmen haben nicht mehr als zehn Beschäftigte, $40 \%$ der Betriebe sogar nur ein oder zwei Beschäftigte. Insbesondere für Kleinbetriebe ist eine hohe Fluktuationsrate typisch, sie liegt bei rund $40 \%$. Insgesamt ist die betriebliche Bindung der Beschäftigten daher eher schwach ausgeprägt, was auch mit einer wenig ausgeprägten Berufsfachlichkeit der Beschäftigten zusammenhängen dürfte. Es ist davon auszugehen, dass im Gastgewerbe zukünftig immer weniger einschlägig beruflich Qualifizierte arbeiten werden: Im Zeitraum 2007-2010 ist die Zahl der Auszubildenden in den sechs wichtigsten Berufen der Branche um $20 \%$ zurückgegangen. Allein im Jahr 2010 befanden sich 10.000 junge Menschen weniger als ein Jahr zuvor im Gastgewerbe in einer Ausbildung. Es liegt die Vermutung nahe, dass die abnehmende Attraktivität des Gastgewerbes als Ausbildungsbranche mit den schlechten Perspektiven für eine Existenz sichernde Erwerbsarbeit in Verbindung steht.

Die kleinbetriebliche Struktur, die hohe Fluktuation und ebenso der hohe Anteil von Anlerntätigkeiten, z. B. als Hotelpage, Türsteher oder Hilfskraft im Küchen- und Restaurantbereich, bringen es mit sich, dass Mindeststandards in der Beschäftigung von Beschäftigten selbst nicht eingefordert werden. Wer mit den Bedingungen nicht zufrieden ist, geht zum nächsten Betrieb oder sucht sich eine Beschäftigung in einer anderen Branche. Gegenwärtig sind keine Anzeichen zu erkennen, dass der instabilen Beschäftigungssituation z. B. durch starke tarifvertragliche Regelungen etwas entgegengesetzt wird. Vielmehr unterliegen nur wenige Arbeitgeber im Gastgewerbe, insbesondere im Gaststättenbereich (Kneipen und Restaurants), der formalen Tarifbindung, und selbst wenn diese vorliegt, wird sie oftmals unterlaufen. Auch durch die Möglichkeit von OTMitgliedschaften, ${ }^{\mathbf{0}}$ die von Arbeitgeberverbänden in mehr als der Hälfte der 18 Tarifbezirke angeboten werden, hat die Tarifbindung und damit die Durchsetzung von tariflich fixierten Standards abgenommen.

Diese branchenspezifischen Rahmenbedingungen sind ein günstiger Nährboden dafür, dass geltende Rechte im Minijob nicht eingehalten werden. Geringfügig Beschäftigte, also knapp die Hälfte aller Beschäftigten im Gastgewerbe, erhalten in der Regel keinen Tariflohn und kein Urlaubs- und Weihnachtsgeld. Selbst der gesetzliche Urlaubsanspruch sowie die Entgeltfortzahlung im Krankheitsfall werden in der Regel weder von den Arbeitgebern gewährt noch von den Beschäftigten eingefordert. Dies geschieht bei den Beschäftigten oftmals aus Unwissenheit, teils aus einer fehlenden Bindung zum Betrieb. Bei den Arbeitgebern scheint sich kein Unrechtsbewusstsein einzustellen, nicht zuletzt, weil die Einstellung vorherrscht, der Minijob wäre eine Art Aushilfsarbeitsverhältnis. Tarifliche und gesetzliche Normen kommen in der geringfügigen und der sozialversicherungspflichtigen Beschäftigung also zur „selektiven“ Anwendung.

Schwarzarbeit ist im Gastgewerbe, vor allem in der Kleinund Kleinstgastronomie, stark verankert. Die 2003 vom Gesetzgeber initiierte Förderung der Minijobs hat die Schwarzarbeit nicht zurückgedrängt. Schwarzarbeit wird stattdessen von vielen nun als weniger riskant angesehen: Der Wegfall des Stundenlimits für Minijobs hat dazu geführt, dass Beschäftigte oftmals nur noch als Minijobber angemeldet sind, tatsächlich jedoch weit über die maximale Ver-

(1) Das Kürzel „OT-Mitgliedschaften“ steht für Mitgliedschaften im Arbeitgeberverband ohne Tarifbindung. 
dienstgrenze für Minijobs verdienen und entsprechend lange dafür arbeiten. Die Differenz wird „schwarz“ ausbezahlt. Bei Kontrollen der Beamten der Finanzkrontolle Schwarzarbeit (FKS) entsteht nach uns vorliegenden Informationen das Problem, in der konkreten Prüfsituation den Nachweis anzutreten, dass die angetroffenen Beschäftigten schwarz arbeiten, da sie ja als Minijobber angemeldet sind. Da es keine Stundenbindung gibt, sind vorhandene Unterlagen wie z. B. Dienstpläne und Arbeitszeitnachweise oftmals unzureichend, um Schwarzbeschäftigung nachzuweisen.

Die dargstellte Entwicklung hat dramatische Auswirkungen auf die Beschäftigungssituation im Gastgewerbe. Inzwischen ist das Gastgewerbe die Branche in Deutschland, die mit 8,7\% den höchsten Anteil an sogenannten Aufstockern aufweist, also Beschäftigten, die zusätzlich zu ihrem Einkommen Arbeitslosengeld II beziehen. Die steigende Zahl der Minijobs im Nebenjob muss auch als Indiz dafür gewertet werden, dass sich die Einkommenssituation im Haupterwerb zunehmend prekärer darstellt und die Menschen gezwungen sind, ein zweites oder gar drittes Arbeitsverhältnis einzugehen.

\section{Schlussfolgerungen: Sozialversicherungspflicht ab der ersten Stunde}

Im Bäckerhandwerk und im Gastgewerbe hat das starke Wachstum von Minijobs den Druck auf Beschäftigungsstandards enorm erhöht. Der Minijob steht in der betrieblichen Praxis für ein Beschäftigungsverhältnis, in dem der flexible Einsatz je nach Arbeitsanfall, das Unterlaufen tariflicher Standards, das Vorenthalten sozialer Rechte wie Urlaub oder Entgeltfortzahlung im Krankheitsfall und niedrige Löhne mehr oder weniger Normalität sind. Diese Praxis geht weder mit einem Unrechtsbewusstsein seitens der Arbeitgeber einher, noch ist zu beobachten, dass Beschäftigte im Minijob - sei es als Hauptbeschäftigung oder im Nebenjob - die ihnen zustehenden Rechte aktiv einfordern. Das hat nicht nur negative Folgen für die materielle und soziale Sicherung von immer mehr Beschäftigten - ein Stichwort ist hier Altersarmut. Mit Zunahme der Minijobs wird es immer schwieriger, Existenz sichernde und sozialversicherungspflichtige Beschäftigung in den beiden Branchen zu halten. Der Minijob hat seit der Neuregelung im Jahre 2003 zu einer massiven Verschlechterung der Beschäftigungsbedingungen daher entscheidend beigetragen.

Welche Schritte sind notwendig, um diese Abwärtsentwicklung zu stoppen?

(1) Zunächst ist ein branchenübergreifender gesetzlicher Mindestlohn einzuführen, der die Löhne der Beschäftigten nach unten absichert. Eine branchenbezogene Mindestlohnregelung als Zwischenschritt scheint für das Gastgewerbe nicht realisierbar, da sich der Deutsche Hotel- und Gaststättenverband (DEHOGA) klar gegen eine entsprechende Regelung ausgesprochen und zudem durch eine Ausweitung der OT-Mitgliedschaft dafür gesorgt hat, dass tarifliche Standards noch stärker unter Druck geraten sind. Auch im Backgewerbe ist bundesweit eher eine geringe Tarifbindung zu verzeichnen. Erste Schritte sind jedoch gemacht, denn die NGG verzeichnet einen langsamen, aber ständigen Anstieg des Organisationsgrades, und erste Tarifabschlüsse in Ländern, die schon seit Jahren keine Tarifbindung mehr in dieser Branche hatten, konnten unterzeichnet werden.

(2) Ferner wird bei zukünftigen Tarifabschlüssen darauf zu achten sein, dass Teilzeitbeschäftigte entsprechend ihrer Arbeitszeit anteilig tarifliche Leistungen wie Mehrarbeitszuschläge, Urlaubsgeld oder Jahressonderzuwendungen erhalten.

(3) Und schließlich zeigt die betriebliche Praxis den offensichtlichen Reformbedarf in der geringfügigen Beschäftigung: Die Mindestforderung ist eine (Wieder)Einführung einer verbindlichen gesetzlichen Höchststundenzahl in der geringfügigen Beschäftigung. Ohne eine Höchststundenzahl wird auch zukünftig nicht verhindert werden können, dass sich der Einsatz von Beschäftigten im Minijob nach den Kundenströmen richtet, mit der Folge von vielen "grauen“ Stunden, die zu sehr niedrigen effektiven Stundenlöhnen führen. Grundsätzlich aber sind soziale Sicherungslücken und Armut von immer mehr Beschäftigten im Back- und Gastgewerbe vorprogrammiert, wenn ein wachsender Teil der Beschäftigten mangels sozialversicherungspflichtiger Beschäftigungsverhältnisse nur noch im Minijob Arbeit findet und damit keine eigenständigen Ansprüche an die sozialen Sicherungssysteme bei geringem Einkommen erwirbt. Es sind also wieder mehr sozialversicherungspflichtige Beschäftigungsverhältnisse zu schaffen und der Anteil von Minijobs muss reduziert werden. Darum lautet die Forderung der NGG, die Sozialversicherungspflicht ab der ersten Stunde einzuführen, was auf die steuer- und abgabenrechtliche Gleichbehandlung aller Beschäftigungsverhältnisse zielt.

\section{AUTOREN}

KARSTEN ROTHE, Leiter derTarifabteilung der Gewerkschaft Nahrung-Genuss-Gaststätten (NGG)-Hauptverwaltung.

karsten.rothe@ngg.net

PETER STÖRLING, Wirtschaftsgruppenleiter Brot- und Backwarenindustrie, NGG-Hauptverwaltung.

peter.stoerling@ngg.net

GUIDO ZEITLER, Wirtschaftsgruppenleiter Hotel- und Gaststättengewerbe, NGG-Hauptverwaltung.

guido.zeitler@ngg.net 